\title{
Nanofluid Flow Comprising Gyrotactic Microorganisms through a Porous Medium
}

\author{
S. Ahmad ${ }^{1}$, M. Ashraf ${ }^{1 \dagger}$ and K. Ali ${ }^{2}$ \\ ${ }^{I}$ Centre for Advanced Studies in Pure and Applied Mathematics, Bahauddin Zakariya University, Multan \\ 60800, Pakistan \\ ${ }^{2}$ Department of Basic Sciences and Humanities, Muhammad Nawaz Sharif University of Engineering and \\ Technology, Multan 60000, Pakistan
}

†Corresponding Author Email: muhammadashraf@bzu.edu.pk

(Received October 7, 2019; accepted February 3, 2020)

\begin{abstract}
Researchers have significantly contributed to heat transfer field and always made out much effort to find new solutions of heat transfer augmentation. Among numerous methods which have been employed to reinforce the thermal efficiency of energy systems, one is the dispersal of gyrotactic microorganisms in commonly used nanofluids. Another way to improve the thermal efficiency is the utilization of the porous media. The present work deals with the study of nanofluid flow comprising gyrotactic microorganisms with allowance for chemical reaction through a porous medium past a stretching sheet. The nonlinear coupled ODEs are obtained after applying the persuasive tool of similarity transformation on governing model PDEs and then undertook numerically by exploiting the SOR (Successive over Relaxation) parameter method. The outcomes of assorted parameters for the flow are surveyed and discussed through graphs and tables. A comparison is correlated with the previously accomplished study and examined to be in an exceptional agreement. The culminations designate that the bioconvection Peclet number and the microorganisms concentration enhance the density of the motile microorganisms. The chemical reaction phenomenon downturns the concentration and enhances the mass transfer rate on sheet surface. The insertion of the gyrotactic microorganisms in the suspensions is widely used in the bio-microsystems. Examples include biotechnology (in order to enhance the transport phenomenon of heat and mass), enzyme biosensor and microfluidics devices like microvolumes and bacteria powered micromixers. The gyrotactic microorganisms also improve nanofluid stability. Microbial-enhanced oil recovery is also application of bioconvection phenomena where nutrients and microorganisms are inserted in oil bearing layer to maintain the variation in permeability. Our results may also be beneficial in improving the proficiency of microbial fuel cells and heat transfer devices.
\end{abstract}

Keywords: Nanofluid; Gyrotactic microorganisms; Chemical reaction; Concentration.

NOMENCLATURE

$\begin{array}{llll}a, c & \text { constants } & N & \begin{array}{l}\text { microorganisms concentration } \\ \text { density of motile microorganisms }\end{array} \\ C_{\infty} & \text { ambient concentration } & N n_{x} & \begin{array}{l}\text { mbient } \\ \text { amcentration }\end{array} \\ C_{f x} & \begin{array}{l}\text { fluid concentration } \\ \text { skin friction }\end{array} & N_{\infty} & \begin{array}{l}\text { Nusselt number } \\ D_{B}\end{array} \\ D_{n} & \text { Brownian motion diffusivity } & N u_{x} & \text { stretching velocity } \\ D_{T} & \text { diffusivity of microorganisms } & u_{w} & \text { ambient velocity } \\ k^{*} & \text { thermophoretic coefficient } & u_{e} & \text { Sherwood number } \\ K_{c} & \text { darcy permeability } & S h_{x} & \text { ambient temperature } \\ m & \text { rate constant of chemical reaction } & T_{\infty} & \text { fluid temperature } \\ & \text { positive constant for nonlinear } & T & \text { cell swimming speed }\end{array}$




$\begin{array}{ll}\psi & \text { stream function } \\ \tau & \text { heat capacity of nanofluid } \\ \rho & \text { fluid density } \\ \mu & \text { dynamic viscosity }\end{array}$

\section{INTRODUCTION}

In new era of research, the novel study of boundary layer nanofluid flow enclosing gyrotactic microorganisms with mass/heat transport over a stretching surface has got a valuable attention among research community and scientists because of its abundant practical employments in various fields of science and bio-technology. Nanofluids play a momentous role in the mechanism of heat transfer. The typical base fluids e.g. water, ethylene glycol and oil have low capacity to enhance the heat transfer rate. However, this complex scenario was resolved by assorting the tiny sized solid fragments in the base fluids. It was basically proposed by Choi (1995) that the tendency of the base fluids to embellish the thermal properties can be more effective by adding nanoparticles in these fluids. By using the same idea, researchers (Hayat et al. 2014; Pal, 2008; Pal and Saha, 2016; Makinde and Ogulu, 2008; Pal, 2003) extended this work towards an inclusive review. Nanofluids are spawned by dispersion of nanoparticles along with base fluids and these fluids are the amalgamations of suspended nano sized pieces $(1-100 \mathrm{~nm})$ in base fluids. The constituents of nanoparticles may contain metals $(\mathrm{Fe}, \mathrm{Al}, \mathrm{Cu}, \mathrm{Ag}, \mathrm{Au})$, carbides $(\mathrm{SiC})$, nitrides $(\mathrm{SiN}$, $\mathrm{AlN})$ and oxides $\left(\mathrm{CuO}, \mathrm{Al}_{2} \mathrm{O}_{3}, \mathrm{SiO}_{2}\right)$. With improved and enhanced thermal mechanism, nanofluids have massive applications including microwave tubes, microelectronic chips, car AC, fuel cells, hybrid power engines, grinder machines, drag reduction, refrigerator, pharmacology, tumor and cancer therapy, gas recovery of boiler exhaust fuel, in supersonic and ultrasonic field, high power lasers, welding cooling, vehicle engine cooling, nuclear system cooling and thermal storage capacity.

The problems of nanofluids have been examined theoretically, experimentally and numerically by different authors. Kumar and Varma (2018) interrogated the hydro-magnetic flow of nanofluid through a porous medium past an extending sheet with variable wall thickness in the existence of Brownian motion. It was found that the heat diffusion increases due to strong magnetic field and it causes the devaluation in heat transfer rate. Stagnation point nanofluid mixed convective electro magneto hydrodynamics (EMHD) flow through a permeable medium with the impact of joule heating over a stretching sheet was premeditated by Lakshmi et al. (2018). The governing PDEs along with BCs were transformed into ODEs and were settled numerically by exploiting the Keller Box method. Both the electric and magnetic parameter increased the temperature distribution at some specific points in the flow region. The flow of nanofluid along with radiation and heat transport past a stretched sheet
$\eta$
$\theta$
$\tilde{\alpha}$
$v$

embedded in a porous medium with temperature jump and velocity slip was estimated by Zheng et al. (2013). An investigation is accomplished by Mabood et al. (2016) on flow of water based nanofluids ( $\mathrm{Al}_{2} \mathrm{O}_{3}$ and $\mathrm{Cu}$ ) with mass and heat transfer under the impacts of radiation, chemical reaction and viscous dissipation. Due to exclusive physical and thermal properties, the nanofluids are much valuable in the industrial fields e.g. extrusion process of aerodynamics, manufacturing of glass fibers, plastic and rubber sheets manufacturing, metallic plates cooling process, cooling or drying process of papers, cooling process of nuclear reactors and MHD power generators. More recent studies regarding the theory and applications of the nanofluids can be seen in reference articles (Makinde et al. 2018; Saidulu et al. 2019; Hayat et al. 2017; Mabood and Das 2016; Bhatti et al. 2018).

Recently, the intention of scholars is diverted towards flow of gyrotactic microorganisms within nanofluids (Xu and Cui, 2018; Mutuku and Makinde, 2014; Kumar et al. 2017; Zuhra et al. 2018; Hussain et al. 2019). Kuznetsov (2010) commenced the abstract work of bioconvection nanofluid incorporating gyrotactic microorganisms and declared that the self-propelled motile microbes strengthen mixing and avert nanoparticles accumulation in nanofluids. Being a recently discovered phenomenon, occurence of bioconvection is the utmost common event involving the microscopic movement of motile microbes that move up (swim upward) like Oxytactic bacteria, Chlamydomonas nivalis and so on. Bioconvection takes place because the microbes are slightly denser than water and can float causing a very dense volatile state due to the microorganisms array on the upper surface of the water. The premises of this apparent fact may not be the same for all species due to the involvement of some factors such as thermal radiation, chemical reaction, gravity and viscous dissipation that specify the swimming in a certain direction. Ramzan et al. (2017) analyzed the mass and heat transfer in both nanofluid and gyrotactic microorganisms. This study included viscous dissipation with convective boundary conditions, slip effects, and non-linear thermal radiation. It was observed that microorganisms motile density declines with climbing values of the Lewis and the Peclet numbers. In an investigation on nanofluid flow comprising gyrotactic microorganisms under the impact of external magnetic field by Chakraborty et al. (2018), it was found that the heat transport rate, momentum boundary layer thickness and the flux rate of motile microorganisms across the fluid medium diminish with an increment in the magnetic field parameter while the nanoparticle concentration enhances. An effort was made out by Tausif et al. 
(2016) to explore the nanofluid flow containing nanoparticles and microorganisms with multiple slip effects. In this study, it was suggested that by escalating the values of various slip parameters, fluid properties like microorganism flux rate and heat/mass transfer rate can be reduced or improvised to achieve the required quality of the final product in polymer industry. The nanofluid flow with magnetohydrodynamics and gyrotactic microorganisms propagating over a stretching/ shrinking surface was analyzed by Shahid et al. (2018). Non-dimensional variables were employed to simplify the governing equations and then the obtained coupled ODEs were solved numerically by applying "Successive Taylor Series Linearization Method (STSLM)". This research explained how the magnetic field and radiation boost up the temperature and velocity of the fluid. Aziz et al. (2012) worked on the bioconvective nanofluid boundary layer flow and reported that the propagation rate of motile microorganisms is marginally affected by the bioconvection parameters. The steady two dimensional nanofluid flow consisting of both gyrotactic microbes and nanoparticles was observed by Iqbal et al. (2017). Moreover, this study comprised stagnation point flow of circuitously striking nanofluid.

The novelty of this approach is to offer a numerical study of a fluid flow comprising gyrotactic microorganisms and nanoparticles in coexistence of chemical reaction towards a sheet. The sheet was stretched nonlinearly. We assume an electrically conducting two-dimensional and steady nanofluid flow enclosing gyrotactic microbes. Momentum, thermal and species boundary layers across the flow region are shown in the flow sketch figure.

Acharya et al. (2016) examined that the motile microorganisms density significantly strengthens with the Lewis and bioconvective Peclet numbers. It is premeditated by khan et al. (2019) that the Sherwood number, density number of motile microorganisms, Nusselt number and skin friction escalate the nanofluid flow containing gyrotactic microorganisms. The flow was taken around a truncated cone. The stratified bioconvective nanofluid flow was considered by Alsaedi et al. (2017). A comprehensive review of bioconvection nanofluid flow over a convectively-heated wedge together with gyrotactic microorganisms in a Darcy-Brinkman porous medium was presented by Zaib et al. (2018). They adopted the Keller box method for numerical simulation and spotted that the enlarging values of Péclet number tend to decrease the motile microorganisms concentration. Various interesting characteristics like nonlinear radiation, gyrotactic microorganisms and Arrhenius energy activation in the flow of electrically conducted Maxwell nanofluid were explored by Waqas et al. (2019). In the available literature, no work on the numerical simulation of nanofluid flow comprising gyrotactic microorganisms and chemical reaction through porous medium past a nonlinear stretching sheet using our proposed method (SOR) has been found, and it makes our work distinguish from previous ones.

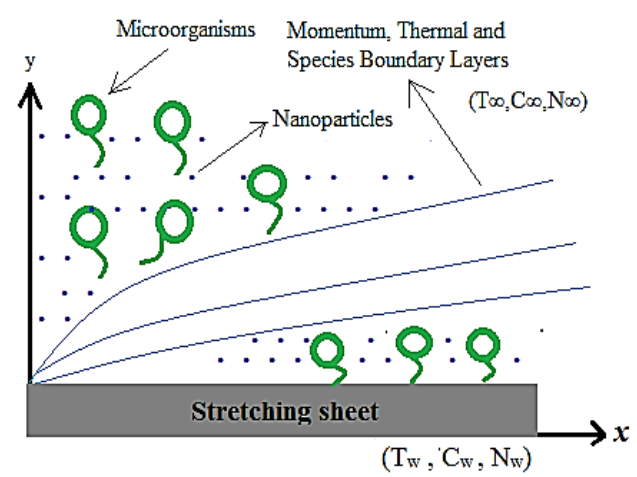

Sketch of the Flow Problem.

We mention some recent theoretical and experimental studies. The correlation of experiments and calculations is a usual practice in developing and understanding new materials (nano particles). Morarji and Gurjar (2019) addressed the motive for the deactivation of a copper catalyst. Their study describes the pathway of an outer-sphere singleelectron. Zhou et al. (2019) established a theory for modeling the photomechanical response of a material, and then expressed an experimental procedure for auxiliary measurements of thermal expansion and laser heating. An experimental and theoretical study on molecular dynamics calculation and density functional theory for electrolyte materials and electrode in sodium-ion batteries was briefly reviewed by Watanabe et al. (2019).

All types of fluid flows through porous media usually exists in scientific and technological fields and its theory has been, successfully, used in the fields such as geothermal and petroleum engineering, micro machines, chemical industry, mineral engineering, soil mechanics and so on. The main purpose of studying this paper is to examine the novel results, achieved during the analysis of nanofluid flow through a porous medium containing nanoparticles and gyrotactic microbes.

The manuscript is organized as follows. The mathematical aspect of the problem in terms of a system of PDEs is presented in the section 2 (physical model), whereas the numerical method employed has been described in section 3. Section 4 is devoted to understand the physical meaning of the numerical results obtained in the graphical and tabular form. Pertinent results, obtained under various situations arising by varying the governing parameters, are discussed and represented through graphs and tables in this section. Finally, the summary with concluding remarks is presented in section 5 .

\section{DESCRIPTION OF PHYSICAL MODEL}

Let, we assume an electrically conducting twodimensional and steady nanofluid flow through porous medium enclosing both the gyrotactic microbes and nanoparticles by taking the impact of 
chemical reaction. $u_{\boldsymbol{e}}(x)=a x^{m}$ is to be taken as ambient velocity and that of $u_{w}(x)=c x^{m}$ is stretching velocity, where $m$ is positive constant other than one (due to nonlinear stretching sheet). In addition, $N_{w}, C_{w}$ and $T_{w}$ are the concentration of the microorganisms, concentration and temperature of fluid at sheet surface, schematically shown in Fig. 1.

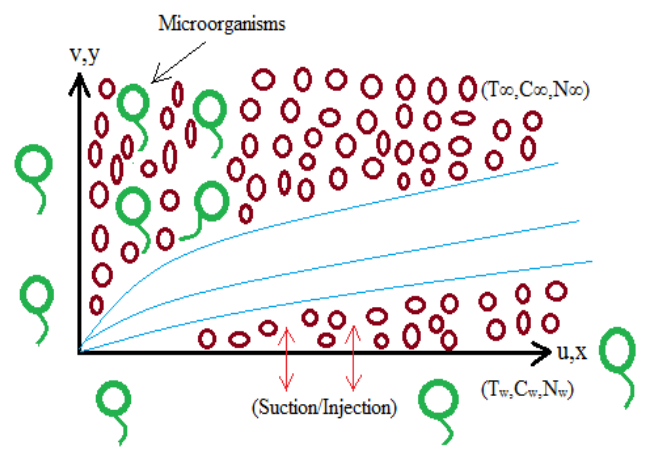

Fig. 1. Physical structure of the Problem.

Following (Aziz et al. 2012; Shahid et al. 2018), the model boundary layer equations under these assumptions, in case of porous medium can be formulated as:

$\frac{\partial v}{\partial y}+\frac{\partial u}{\partial x}=0$

$u \frac{\partial u}{\partial x}+v \frac{\partial u}{\partial y}=v \frac{\partial^{2} u}{\partial y^{2}}+u_{e} \frac{\partial u_{e}}{\partial x}+\frac{\mu}{\rho k^{*}}\left(u_{e}-u\right)$

$u \frac{\partial T}{\partial x}+v \frac{\partial T}{\partial y}=\frac{\tau D_{T}}{T_{\infty}}\left(\frac{\partial T}{\partial y}\right)^{2}+\tilde{\alpha} \frac{\partial^{2} T}{\partial y^{2}}+\tau D_{B} \frac{\partial C}{\partial y} \frac{\partial T}{\partial y}$

$v \frac{\partial C}{\partial y}+u \frac{\partial C}{\partial x}=D_{B} \frac{\partial^{2} C}{\partial y^{2}}-K_{C}\left(C-C_{\infty}\right)+\frac{D_{T}}{T_{\infty}} \frac{\partial^{2} T}{\partial y^{2}}$

(4)

$v \frac{\partial N}{\partial y}+u \frac{\partial N}{\partial x}+\frac{1}{C_{w}-C_{\infty}} \frac{\partial}{\partial y}\left[N\left(\frac{\partial C}{\partial y}\right)\right] b W_{c}=D_{n} \frac{\partial^{2} N}{\partial y^{2}}$

where $\mathrm{x}$ and $\mathrm{y}$ be the coordinates of the axes along and normal to the sheet with $u$ and $\mathrm{v}$ being the respective velocity components in the $x$ and $y$ directions, respectively.

The respective $\mathrm{BCs}$ are:

$u=c x^{m}, N=N_{w}, C=C_{w}, T=T_{w}, v=v_{w}(x)$ at $y=0$

$u=a x^{m}, N=N_{\infty}, C=C_{\infty}, T=T_{\infty} \quad$ at $y \rightarrow \infty$

$v_{w}$ is mass flux which corresponds to suction $\left(v_{w}>0\right)$ and injection $\left(v_{w}<0\right)$.

Following similarity transformations are introduced along with stream function to transmute the governing PDEs into ODEs.

$$
\begin{aligned}
& \psi=\sqrt{a x^{m+1} v} f(\eta), \eta=\sqrt{\frac{a}{v} x^{m-1}} y, G(\eta)=\frac{N-N_{\infty}}{N_{w}-N_{\infty}}, \\
& \phi(\eta)=\frac{C-C_{\infty}}{C_{w}-C_{\infty}}, \theta(\eta)=\frac{T-T_{\infty}}{T_{w}-T_{\infty}}
\end{aligned}
$$

Entreating these variables in Eqs. (2)-(5), we acquire the following set of equations:

$$
\begin{aligned}
& f^{\prime \prime \prime}+P_{0}\left(1-f^{\prime}\right)=m\left(-1+f^{\prime 2}\right)-\frac{1+m}{2} f f^{\prime \prime} \\
& \frac{1}{\operatorname{Pr}} \theta^{\prime \prime}+N_{b} \phi^{\prime} \theta^{\prime}+\frac{1+m}{2} f \theta^{\prime}+N_{t} \theta^{2}=0 \\
& \phi^{\prime \prime}-L e R_{C} \phi=-\frac{N_{t}}{N_{b}} \theta^{\prime \prime}-\frac{1+m}{2} L e f \phi^{\prime} \\
& G^{\prime \prime}+\frac{1+m}{2} S c f G^{\prime}=\operatorname{Pe}\left[\phi^{\prime} G^{\prime}+(G+\Omega) \phi^{\prime \prime}\right]
\end{aligned}
$$

The interrelating $\mathrm{BCs}$ are:

$$
\begin{aligned}
& \eta=0: \quad f=S=1, f^{\prime}=\alpha, \phi=1, \theta=1 \\
& \eta \rightarrow \infty: \quad \phi \rightarrow 0, f^{\prime} \rightarrow 1, \theta \rightarrow 0, G \rightarrow 0
\end{aligned}
$$

where $\alpha$ is the stretching of sheet parameter, $S$ is suction/injection and the other parameters involved in the system of Eqs. (9)-(12) are:

$$
\begin{aligned}
& P_{0}=\frac{v x}{k^{*} u_{e}}, N_{b}=\frac{\tau D_{B}\left(C_{w}-C_{\infty}\right)}{v}, \operatorname{Pr}=\frac{v}{\alpha}, \\
& N_{t}=\frac{\tau D_{T}\left(T_{w}-T_{\infty}\right)}{v T_{\infty}}, L e=\frac{v}{D_{B}}, R_{c}=\frac{2 K_{c} x}{u_{e}}, \\
& \Omega=\frac{N_{\infty}}{N_{w}-N_{\infty}}, S c=\frac{v}{D_{n}}, P e=\frac{b W_{c}}{D_{n}} .
\end{aligned}
$$

where $P_{0}$ signifies the porosity parameter, $N_{b}$ refers to the parameter of Brownian motion, $\mathrm{Pr}$ exemplifies the Prandtl number, $N_{t}$ denotes the thermophoresis parameter, Le stands for Lewis number, $R_{C}$ describes the chemical reaction parameter, $\Omega$ is recognized as motile microorganisms parameter, Sc conveys the Bioconvection Schmidt number and $P e$ indicates the Peclet number. The quantities of engineering interest in dimensionless form like local Sherwood number, local density number of motile microorganisms, skin friction and Nusselt number may be defined as:

$$
\begin{aligned}
& \operatorname{Re}_{x}^{-\frac{1}{2}} S h_{x}=-\phi^{\prime}(0), \quad \operatorname{Re}_{x}^{-\frac{1}{2}} N n_{x}=-G^{\prime}(0), \\
& \operatorname{Re}_{x}^{\frac{1}{2}} C_{f x}=f^{\prime \prime}(0), \quad N u_{x} \operatorname{Re}_{x}^{-\frac{1}{2}}=-\theta^{\prime}(0) .
\end{aligned}
$$

whereas $\operatorname{Re}_{x}=U_{e^{x}} / v$ denotes the local Reynolds 
number.

\section{NUMERICAL APPROACH}

The nonlinear Eqs. (9)-(12) have been solved by using finite difference discretization. Some complications are found in the numerical solution of the boundary value problems comprising boundary layer equations, if the outer boundary conditions are positioned at infinity. The greater estimation values of the independent variable may numerically approximate the infinity in the test integration. But there is no idea or any general rule to estimate these values. Choosing a large estimation value for the independent variable may diverges the solution or converges very slowly. Similarly, too small value may not provide the required precision in the trial integration. However, exploiting the SOR method one can overcome such type of difficulties. Unlike other numerical techniques, SOR method is a well renowned scheme to find the approximate solutions of nonlinear differential equations with very quick convergence. Initially and almost simultaneously Frankel (1950) and Young (1950) entrenched the theory of SOR. We construct numerical algorithm of our problem by using finite difference technique (Syed et al. 1997). As prescribed by Chamkha (2000) and Ashraf and Wehgal (2012), we recede the order of Eq. (9) by modifying

$$
s=f^{\prime}=\frac{d f}{d \eta}
$$

so that we have to solve the nonlinear Eqs. (9)-(12) in the form:

$$
\begin{aligned}
& s^{\prime \prime}+P_{0}(1-s)=m\left(-1+s^{2}\right)-\frac{1+m}{2} f s^{\prime} \\
& \frac{1}{\operatorname{Pr}} \theta^{\prime \prime}+N_{b} \phi^{\prime} \theta^{\prime}+\frac{1+m}{2} f \theta^{\prime}+N_{t} \theta^{2}=0 \\
& \phi^{\prime \prime}-L e R_{C} \phi=-\frac{N_{t}}{N_{b}} \theta^{\prime \prime}-\frac{1+m}{2} L e f \phi^{\prime} \\
& G^{\prime \prime}+\frac{1+m}{2} S c f G^{\prime}=\operatorname{Pe}\left[\phi^{\prime} G^{\prime}+(G+\Omega) \phi^{\prime \prime}\right]
\end{aligned}
$$

with $\mathrm{BCs}$

$$
\begin{aligned}
& f(0)=1, s(0)=\alpha, s(\infty)=1, \theta(0)=1, \theta(\infty)=0, \\
& G(0)=1, G(\infty)=0, \phi(0)=1, \phi(\infty)=0 .
\end{aligned}
$$

The finite differences for the derivatives $s_{i}^{\prime}, s_{i}^{\prime \prime}, G_{i}^{\prime}, G_{i}^{\prime \prime}, \theta_{i}^{\prime}, \theta_{i}^{\prime \prime}, \phi_{i}^{\prime}$ and $\phi_{i}^{\prime \prime}$ are given in the appendix (please see Eqs. (21)-(25) in appendix).

The nonlinear algebraic system obtained from Eqs. (16)-(19) after using the finite differences is solved repetitively, with respect to the BCs (20), by the SOR method. In order to make the better exactness of the numerical solution and for an appropriate decision of the estimations of the framework, we ratify the following criteria.
- To generate the next approximations $\widehat{s}(k+1)$, $\hat{G}^{(k+1)}, \hat{\theta}^{(k+1)}$ and $\hat{\phi}^{(k+1)}$ the solution of Eqs. (21)-(24) (see appendix) is determined with the help of SOR method subject to the last eight conditions in (20).

- New guess $\widehat{f}^{(k+1)}$ for the solution of Eq. (15) is processed, subject to the first condition given in (20), by Simpson's rule which is then utilized for $\widehat{s}^{(k+1)}$ in Eq. (21).

- Initially, we take the basic grid say $h \in[0, H]$ to build the order of precision of the discrete problem.

- For the convergence test, ${ }_{s}(k+1)$, $\hat{\theta}^{(k+1)}, \widehat{G}^{(k+1)}, \hat{\phi}^{(k+1)}$ and $\hat{f}^{(k+1)}$ are calculated along with $\widehat{s}^{(k)}, \hat{\theta}^{(k)}, \widehat{G}^{(k)}, \hat{\phi}^{(k)}$ and $\hat{f}^{(k)}$.

The iterative process is continued as far as:

$$
\begin{aligned}
& \max \left(\left\|\hat{G}^{(k+1)}-\hat{G}^{(k)}\right\|_{L_{2}},\left\|\hat{\phi}^{(k+1)}-\hat{\phi}^{(k)}\right\|_{L_{2}}\right)<T O L_{\text {iter }} \\
& \max \left(\left\|\hat{s}^{(k+1)_{-}}(k)\right\|_{L_{2},}, \quad\left\|\hat{\theta}^{(k+1)}-\hat{\theta}^{(k)}\right\|_{L_{2}}\right)<T O L_{i t e r} \\
& \max \left(\| \hat{f}^{(k+1)_{-}}\left(\hat{f}^{(k)} \|_{L_{2}}\right)\right)<\text { TOL iter }
\end{aligned}
$$

For all the calculations performed here, the value of TOL $L_{\text {iter }}$ is taken at least $10^{-11}$. A comparison is correlated with the previously accomplished results in terms of numerical data (see Table 1). The assessment is found to be in an exceptional agreement and it ensures the accuracy of our numerical procedure.

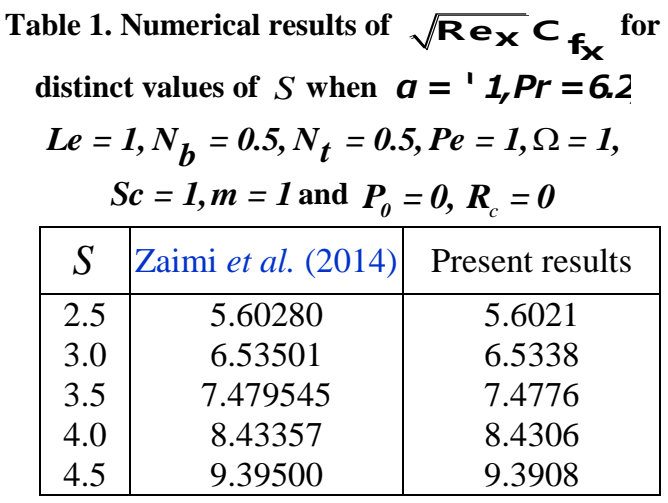

\section{RESULTS AND DISCUSSION}

The nonlinear governing Eqs. (16)-(19) are solved numerically subject to the $\mathrm{BCs}(20)$ by means of a 
Table $2 C_{f x}, N u_{x}, S h_{x}$ and $N n_{x}$ for various $m$ and $P_{0}$

\begin{tabular}{|c|c|c|c|c|c|c|}
\hline$m$ & $C_{f x}$ & $N u_{x}$ & $S h_{x}$ & $N n_{x}$ & $P_{0}$ & $C_{f x}$ \\
\hline 1 & 1.1180 & -1.7496 & -1.9773 & -2.8167 & 11 & 2.3414 \\
2 & 1.5227 & -2.6370 & -2.0510 & -3.6408 & 22 & 2.9375 \\
3 & 1.8842 & -3.5223 & -2.1358 & -4.4548 & 33 & 3.4169 \\
4 & 2.2211 & -4.4060 & -2.2294 & -5.2644 & 44 & 3.8282 \\
5 & 2.5419 & -5.2887 & -2.3296 & -6.0719 & 55 & 4.1934 \\
\hline
\end{tabular}

Table $3 N u_{x}$ for various $\operatorname{Pr} \& N_{t}$

\begin{tabular}{|c|c|c|c|}
\hline $\operatorname{Pr}$ & $N u_{x}$ & $N_{t}$ & $N u_{x}$ \\
\hline 1 & -1.7760 & 0.0 & -5.9286 \\
3 & -3.1384 & 0.3 & -3.7795 \\
5 & -3.9588 & 0.6 & -2.4252 \\
7 & -4.5562 & 0.9 & -1.6045 \\
10 & -5.2293 & 1.2 & -1.1059 \\
\hline
\end{tabular}

Table $4 S h_{x}$ for various $N_{b}$, Le \& $R_{c}$

\begin{tabular}{|c|c|c|c|c|c|}
\hline$N_{b}$ & $S h_{x}$ & $L e$ & $S h_{x}$ & $R_{c}$ & $S h_{x}$ \\
\hline 0.2 & -2.1566 & 0.10 & 0.9865 & 1 & -1.0945 \\
0.3 & -2.9252 & 0.15 & 0.8029 & 2 & -1.5474 \\
0.5 & -3.5129 & 0.20 & 0.6346 & 3 & -1.9260 \\
0.8 & -3.8051 & 0.25 & 0.4794 & 4 & -2.2525 \\
1.5 & -3.9600 & 0.30 & 0.3349 & 5 & -2.5408 \\
\hline
\end{tabular}

Table $5 N n_{x}$ for various $S_{c}, P e \& \Omega$.

\begin{tabular}{|c|c|c|c|c|c|}
\hline$S_{c}$ & $N n_{x}$ & $P e$ & $N n_{x}$ & $\Omega$ & $N n_{x}$ \\
\hline 0.2 & -1.41874 & 1.0 & -7.41274 & 1.0 & -2.68754 \\
0.4 & -1.87810 & 1.6 & -9.74881 & 1.5 & -2.98438 \\
0.6 & -2.31917 & 2.2 & -12.08066 & 2.0 & -3.28122 \\
0.8 & -2.74708 & 2.8 & -14.39948 & 2.5 & -3.57806 \\
1.0 & -3.16660 & 3.4 & -16.69895 & 3.0 & -3.87490 \\
\hline
\end{tabular}

finite-difference based technique known as the SOR method, as described in the book by Hildebrand (1978), for a variety of estimated values of the governing parameters e.g. $P_{0}, m, \operatorname{Pr}, N_{b}, N_{t}, L e, R_{c}$, Sc, Pe, $\Omega$ and $\alpha$. The step size of $\eta$ as well as the edge of the boundary layer are balanced in such a way that the velocity $F^{\prime}(\eta)$, temperature $\theta(\eta)$, concentration $\phi(\eta)$ and motile microorganisms density distribution $G(\eta)$ show asymptotic demeanor for various scopes of physical parameters. Some of the parametric values are assumed fixed such as

$$
P_{0}=0.6, R_{c}=1, S c=1.5, P e=0.4, \Omega=0.2
$$

and $\alpha=0.5$ otherwise specified. From Table 2 , it is obvious that the impact of positive constant $m$ and the porosity parameter $P_{0}$ is to escalate the skin friction coefficient while $m$ also strengthens heat and mass transport rates as well as motile microorganisms density. It is noted from Table 3 that the Prandtl number Pr improves the heat transfer rate however $N_{t}$ (thermophoresis parameter) reduces it. The values of the Sherwood number are boost up with rising values of the Brownian parameter $N_{b}$ and the chemical reaction parameter $R_{C}$ while these values diminish with the influence of the Lewis number $L e$, as envisioned in Table 4. Moreover, Table 5 predicts that the bioconvection Schmidt number $S_{C}$, the Peclet number $P e$ and the microorganisms concentration difference parameter $\Omega$ increase the density of the motile microorganisms.

The results reveal that the porous medium enhances the shear stress and that of bioconvection parameters ( $S_{C}, P e, \Omega$ ) enhance the density of the motile microorganisms respectively. It is seen here that heat transfer rate grows with the parameter of Brownian motion and drops with thermophoretic parameter. Also, it is revealed that the movement of the particles is in crisscross manner because of the existence of base fluid in nanoparticles which causes the collision of particles inside the fluid and generates more heat. However, collision due to thermophoretic parameter among the particles produces more and more heat and thus reducing the rate of heat transfer and growing the temperature. 


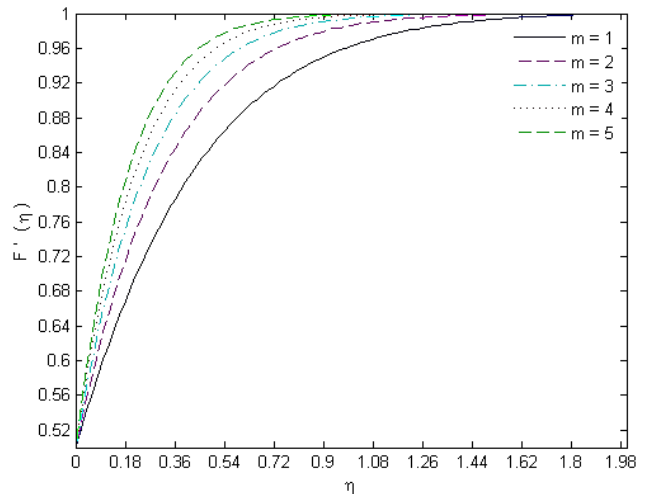

Fig. 2. $F^{\prime}(\eta)$ for various $m$ and

$$
\operatorname{Pr}=3.2, N_{t}=0.3, L e=2, N_{b}=0.3 \text {. }
$$

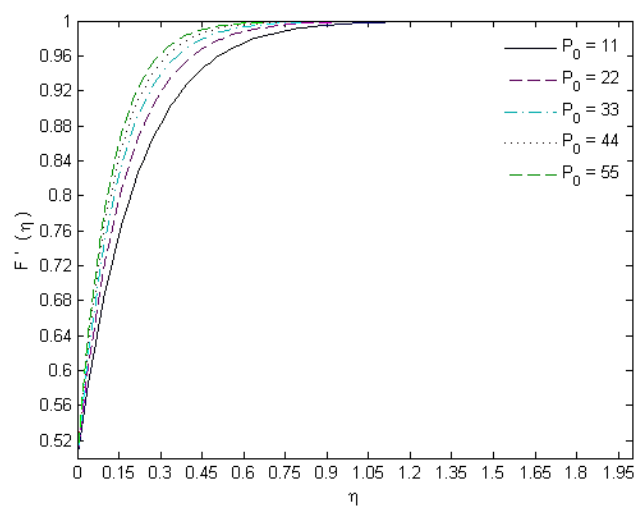

Fig. 4. $F^{\prime}(\eta)$ for various $P_{0}$ and $N_{b}=0.3$,

$$
\operatorname{Pr}=7.2, L e=2, m=2, N_{t}=0.3 .
$$

Figures 2 and 3 describe the demeanor of velocity and motile microorganisms density profiles for multiple values of $m$. The profile $F^{\prime}(\eta)$ rises up whereas the profile $G(\eta)$ across the boundary layer shows a decreasing trend with $m$. The stretching of sheet subsidized to accelerate the flow velocity that can be observed from Fig. 2. Furthermore, $\alpha>0$ denotes the stretching of sheet, $\alpha<0$ corresponds to the shrinking case, $\alpha=1$ describes no boundary layer and $\alpha=0$ is correlated with the planar stagnation point flow.

Figure 4 reveals impact of the porosity parameter $P_{0}$ on streamwise velocity. An increment in $P_{0}$, marginally boosts magnitude of the velocity. It is also observed here from Figs. 5 and 6 that the thermophoresis parameter substantially increases the temperature while an opposite trend, as compared with $N_{t}$, is noted in case of Prandtl number. It is observed here that the effect of $N_{t}$ significantly enhances the nanofluid temperature and hence the thickness of the thermal boundary layer enhances. This anomaly designates that the flow is faster away

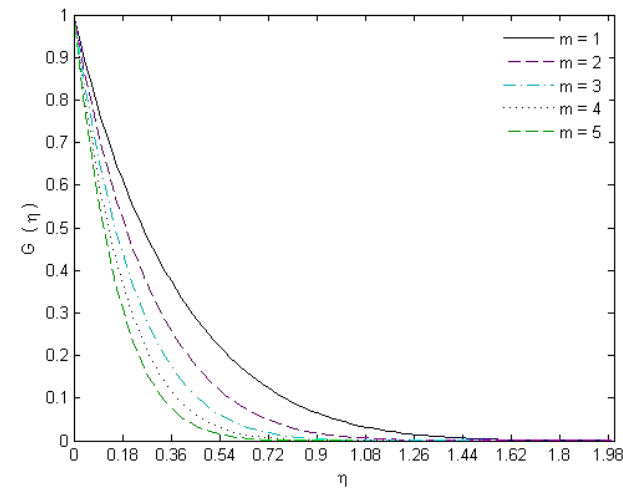

Fig. 3. $\boldsymbol{G}(\eta)$ for various $m$ and

$$
L e=2, N_{t}=0.3, \operatorname{Pr}=3.2, N_{b}=0.3 .
$$

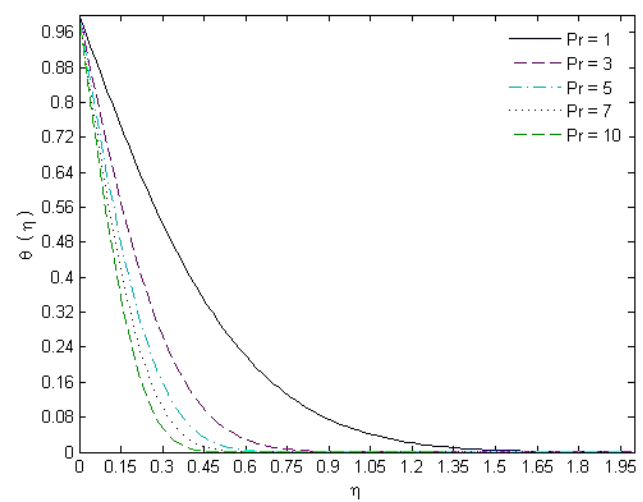

Fig. 5. $\theta(\eta)$ for various $P r$ and $N_{b}=0.3$,

$$
L e=2, N_{t}=0.4, m=3 \text {. }
$$

from the surface and slower near the surface due to thermophoretic force. Subsequently more heated fluid travels away from the surface which tends to increase the temperature. The force induced by the thermophoresis has competency to shift the particles from hotter to cooler area. Furthermore, if the fluid comprehends the high Prandtl number then its temperature drops down.

Figures 7-9 are drawn against the concentration $\phi(\eta)$ for various Lewis number, parameter of Brownian motion and the chemical reaction parameter. The effect of these three parameters is to downturn the concentration profiles. The behavior of the motile microorganisms density profiles for the distinct values of the bioconvection Schmidt number, Peclet number and the microorganism concentration difference parameter have been examined in Figs. 10-12. The microorganisms concentration profiles fall with escalating values of $S c, P e$ and $\Omega$. The cell swimming $W_{c}$ is directly proportional to $P e$ (Peclet number) and inversely proportional to $D_{n}$ (diffusivity of microorganisms). Peclet number is the proportion between the rates of diffusion and advection. So, an enhancement in the bioconvection 


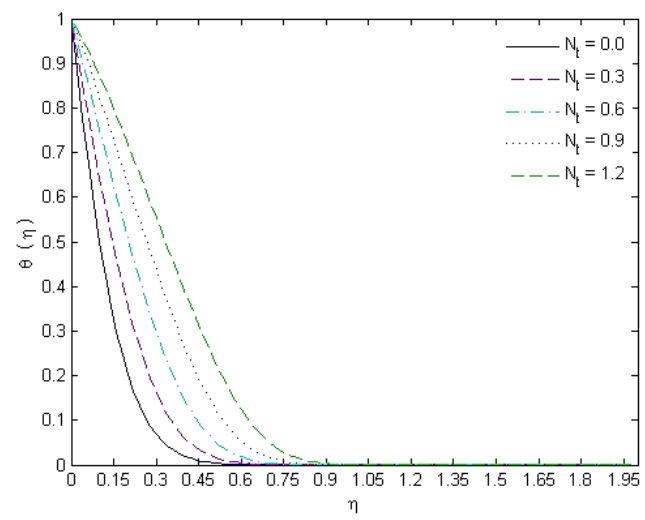

Fig. 6. $\theta(\eta)$ for various $N_{t}$ and $m=2$, $N_{b}=0.3, L e=2, \operatorname{Pr}=7.2$.

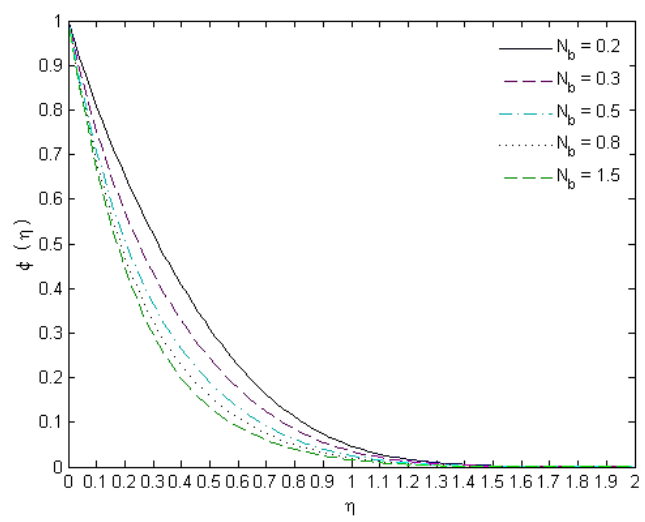

Fig. 8. $\phi(\eta)$ for various $N_{b}$ and $m=2, \operatorname{Pr}=2, L e=2, N_{t}=0.2$.

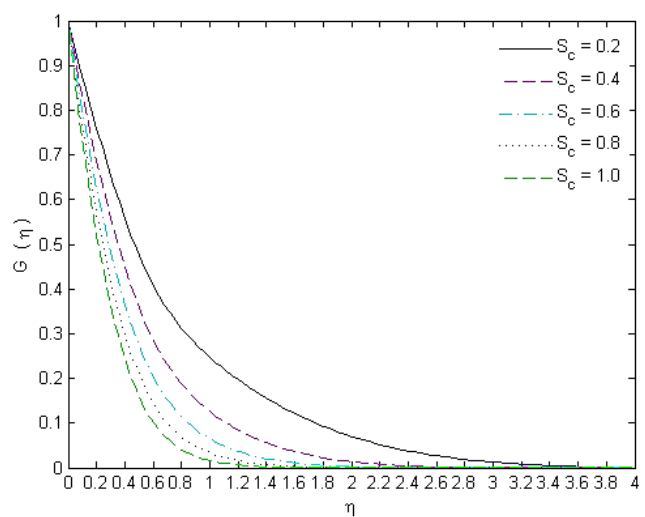

Fig. 10. $G(\eta)$ for various $S c$ and $m=3$,

$$
\operatorname{Pr}=3.2, N_{b}=0.3, L e=2, N_{t}=0.4 \text {. }
$$

$P e$ gives rise to an increment in the advective transport rate as compared to the diffusion, and consequently the flux of the self-swimming microorganisms rapidly rises. Hence growing Peclet number escalates the wall motile microorganisms flux.

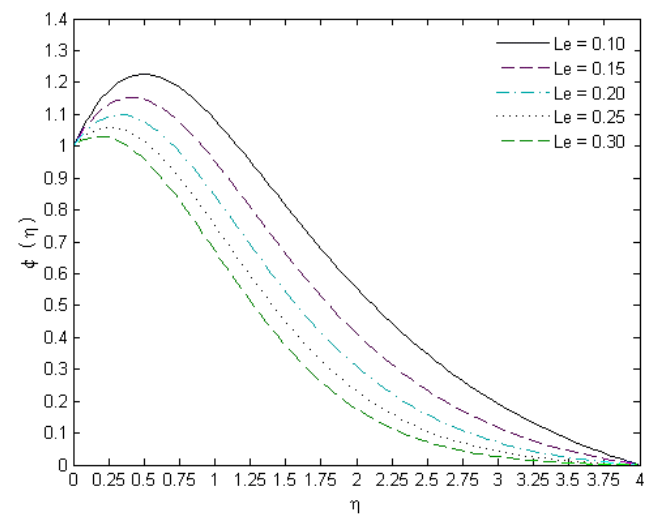

Fig. 7. $\phi(\eta)$ for various $L e$ and

$N_{b}=0.2, \operatorname{Pr}=1, m=2, N_{t}=0.2$.

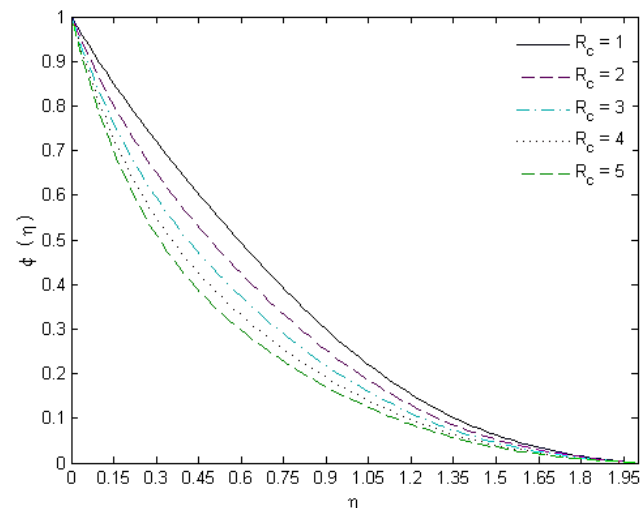

Fig. 9. $\phi(\eta)$ for various $R_{c}$ and $m=2$,

$$
N_{b}=0.3, \operatorname{Pr}=1, \text { Le }=1, N_{t}=0.4 \text {. }
$$

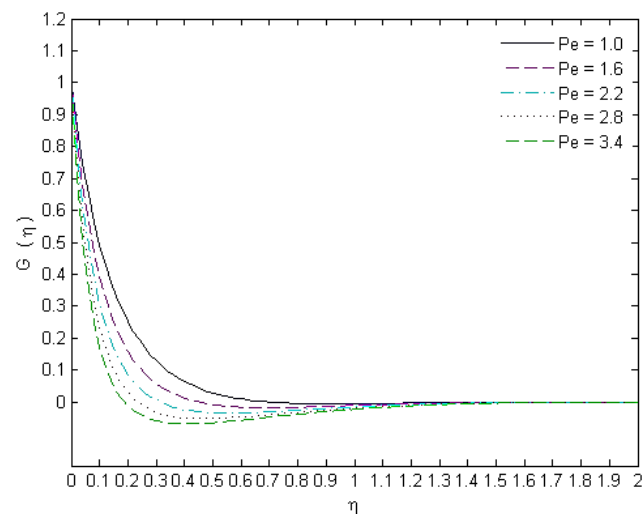

Fig. 11. $G(\eta)$ for various $P e$ and $m=3$,

$$
N_{b}=0.2, L e=2, N_{t}=0.2, \operatorname{Pr}=1 \text {. }
$$

It is noticed that the concentration profile as well as thickness of the concentration boundary layer substantially diminish by expanding the estimations of $L e$. This may occur because of the way that by expanding the estimations of $L e$ there 
is decline in the nanofluid mass diffusivity which lessens the concentration of nanoparticles. Besides, as the bioconvection Le enhances, the rate of viscous diffusion builds which lessens the fluid speed at the sheet surface, and likewise diminishes the density of the motile microorganisms. The bioconvection Peclet number $P e$ builds the swimming rate of the motile microorganisms in regard of the fluid thus the thickness of the microorganisms is decreased close to the surface. The density for motile microorganisms is decreased by escalating the estimation values of the microorganisms parameter. Thereby, one may decide that the bioconvection parameters substantially influence the motile microorganisms transport rate.

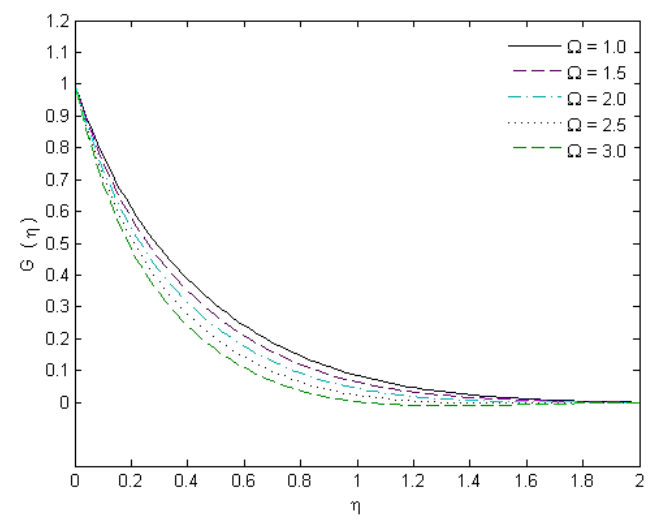

Fig. 12. $G(\eta)$ for various $\Omega$ and $\operatorname{Pr}=1$,

$$
\begin{aligned}
N_{t}=0.4, S c & =0.5, P e=0.2, m=3, \\
N_{b} & =0.3, L e=2 .
\end{aligned}
$$

Now, we mention some limitations and advantages of this study. The assumption of self-similarity (employed in the present work) to reduce the NavierStoke equations from PDEs to ODEs remarkably simplifies the analysis, but there is a need to assure the physical realizable solutions. Therefore, in a subsequent paper, we intend to compare our results with the ones by solving the governing PDEs. But, there is potential of employing nanofluids with microorganisms in several bio-microsystems like chip-shaped microdevices to evaluate toxicity of nanoparticles, the optimization of celluloses production and provocative response of the lung to silica nanoparticles. Besides, fluid flow over a stretching surface has numerous important employments in engineering and technological processes such as manufacturing filaments, polymer extrusion, wind up roller, petroleum manufacturing goods, drawing of plastic films and polymer sheets etc.

\section{SUMMARY AND CONCLUSIONS}

A comprehensive numerical investigation of the nanofluid dynamics through a porous medium taking into account the effects of gyrotactic microorganisms and chemical reaction is introduced. SOR method is employed to acquire the numerical solution against concentration, thermal energy, motile microorganisms density and velocity. The major findings of this article are mentioned below:

- Porous medium enhances the shear stress as well as the flow.

- $\quad$ The enhancement in $S_{c}, P e$ and $\Omega$ causes the increase in microorganisms diffusion rate while it declines the density profiles.

- The large estimations of Peclet number are linked with low diffusivity of microorganisms that causes the reduction in motile microorganisms density profile.

- The Lewis number and the thermophoresis parameter both reduce the mass and heat transfer rates respectively.

\section{APPENDIX}

After using finite differences for $s_{i}^{\prime}, s_{i}^{\prime \prime}, G_{i}^{\prime}, G_{i}^{\prime \prime}, \theta_{i}^{\prime}, \theta_{i}^{\prime \prime}, \phi_{i}^{\prime}$ and $\phi_{i}^{\prime \prime}$, Eqs. (16) - (19) take the form:

$$
\begin{aligned}
& \widehat{s}_{i}=\frac{1}{A_{1}}\left(B_{1} \widehat{s}_{i+1}+C_{1} \widehat{s}_{i-1}+D_{1}\right) \\
& \widehat{G}_{i}=\frac{1}{A_{2}}\left(B_{2} \widehat{G}_{i+1}+C_{2} \widehat{G}_{i-1}-D_{2}\right) \\
& \widehat{\theta}_{i}=\frac{1}{A_{3}}\left(B_{3} \widehat{\theta}_{i+1}+C_{3} \widehat{\theta}_{i-1}+D_{3}\right) \\
& \widehat{\phi}_{i}=\frac{1}{A_{4}}\left(B_{4} \widehat{\phi}_{i+1}+C_{4} \widehat{\phi}_{i-1}+D_{4}\right)
\end{aligned}
$$

Where

$$
\begin{aligned}
& A_{1}=4+2 h^{2} P_{0}+2 h^{2} m \bar{s}_{i}, B_{1}=\frac{1+m}{2} h f_{i}+2, \\
& C_{1}=2-\frac{1+m}{2} h f_{i}, \quad D_{1}=2 h^{2} m+2 h^{2} P_{0} \\
& A_{2}=\frac{8}{\operatorname{Pr}}, B_{2}=\frac{4}{\operatorname{Pr}}+\left(-\widehat{\phi}_{i-1}+\widehat{\phi}_{i+1}\right) N_{b}+(1+m) h f_{i}+ \\
& N_{t} \hat{\theta}_{i+1}-2 N_{t} \hat{\theta}_{i-1}, \quad C_{2}=\frac{4}{\operatorname{Pr}}-N_{b}\left(-\widehat{\phi}_{i-1}+\widehat{\phi}_{i+1}\right), \\
& -(1+m) h f_{i}+N_{t} \hat{\theta}_{i-1}, \quad D_{2}=0, \quad A_{3}=4+2 h^{2} L e R_{c}, \\
& B_{3}=2+\frac{1+m}{2} L e h f_{i}, \quad C_{3}=2-\frac{1+m}{2} L e h f_{i}, \\
& D_{3}=2 \frac{N_{t}}{N_{b}}\left(\widehat{\theta}_{i-1}+\hat{\theta}_{i+1}-2 \widehat{\theta}_{i}\right), A_{4}=4+2 P e \\
& \left(\widehat{\phi}_{i-1}+\widehat{\phi}_{i+1}-2 \widehat{\phi}_{i}\right), B_{4}=2+\frac{1+m}{2} h S c f_{i}-\frac{P e}{2}\left(-\widehat{\phi}_{i-1}+\widehat{\phi}_{i+1}\right), \\
& C_{4}=2-\frac{1+m}{2} h S c f_{i}+\frac{P e}{2}\left(-\widehat{\phi}_{i-1}+\widehat{\phi}_{i+1}\right), \\
& D_{4}=-2 P e \Omega\left(-2 \widehat{\phi}_{i}+\widehat{\phi}_{i+1}+\widehat{\phi}_{i-1}\right) .
\end{aligned}
$$




\section{REFERENCES}

Acharya, N., D. Kalidas and P. K. Kundu (2016). Framing the effects of solar radiation on magneto-hydrodynamics bioconvection nanofluid flow in presence of gyrotactic microorganisms. Journal of Molecular Liquids $222,28-37$.

Alsaedi, A., M. Ijaz Khan, M. Farooq, N. Gull and T. Hayat (2017). Magnetohydrodynamic (MHD) stratified bioconvective flow of nanofluid due to gyrotactic microorganisms. Advanced Powder Technology 28, 288-298.

Ashraf, M. and A. R. Wehgal (2012). MHD flow and heat transfer of micropolar fluid between two porous disks. Applied Mathematics and Mechanics Eng. 33, 51-64.

Aziz, A., W. A. Khan and I. Pop (2012). Free convection boundary layer flow past a horizontal flat plate embedded in porous medium filled by nano-fluid containing gyrotactic microorganisms. International Journal of Thermal Sciences 56, 48.

Bhatti, M. M., M. A. Abbas and M. M. Rashidi (2018). A robust numerical method for solving stagnation point flow over a permeable shrinking sheet under the influence of MHD. Applied Mathematics and Computation 316, 381-389.

Chakraborty, T., D. Kalidas and P. K. Kundu (2018). Framing the impact of external magnetic field on bioconvection of a nanofluid flow containing gyrotactic microorganisms with convective boundary conditions. Alexandria Enggineering Journal 57, 61-71.

Chamkha, A. J. and A. Camille Issa (2000). Effects of heat generation/absorption and thermophoresis on hydromagnetic flow with heat and mass transfer over a flat surface. International Journal of Numerical Methods in Heat and Fluid Flow 10, 432-449.

Choi, S. U. S. (1995). Enhancing thermal conductivity of fluids with nanoparticles. In development and applications of nonnewtonian flow. ASME. FED-vol. 231/MD-vol. $66,99-105$.

Frankel, S. P. (1950). Convergence rates of iterative treatments of partial differential equations, Mathematical Tables and Other Aids to Computation 4, 65-75.

Hayat, T., M. Imtiaza, A. Alsaedib and R. Mansoora (2014). MHD flow of nanofluids over an exponentially stretching sheet in a porous medium with convective boundary conditions. Chinese Physics B 23 (5), 054701.

Hayat, T., M. Waqas, A. Alsaedi, G. Bashir and F. Alzahrani (2017). Magnetohydrodynamic (MHD) stretched flow of tangent hyperbolic nanoliquid with variable thickness. Journal of
Molecular Liquids 229, 178-184.

Hildebrand, F. B. (1978). Introduction to Numerical Analysis. Tata McGraw-Hill Publishing Company: New Delhi.

Hussain, S. A., G. Ali, S. I. A. Shah, S. Muhammad and M Ishaq (2019). Bioconvection model for magneto hydrodynamics squeezing nanofluid flow with heat and mass transfer between two parallel plates containing gyrotactic microorganisms under the influence of thermal radiations. PUJM. 51, 13-36.

Iqbal, Z., Z. Mehmood and E. N. Maraj (2017). Oblique transport of gyrotactic microorganisms and bioconvection nanoparticles with convective mass flux. Physica E 88, 265-271.

Khan, W. A., A. M. Rashad, M. M. M. Abdou, and I. Tlili (2019), Natural bioconvection flow of a nanofluid containing gyrotactic microorganisms about a truncated cone. European Journal of Mechanics B/Fluids $75,133-142$.

Kumar, K. R. V. M. S. S. and S.V. K. Varma (2018). MHD boundary layer flow of nanofluid through a porous medium over a stretching sheet with variable wall thickness: using cattaneochristov heat flux model. JTAM 48 (2), 72-92.

Kumar, R., S. Sood, S. A. Shehzad and M. Sheikholeslami(2017). Numerical modeling of time-dependent bio-convective stagnation flow of a nanofluid in slip regime. Results Physics 7 , $3325-3332$.

Kuznetsov, A. V. (2010). The onset of nanofluid bioconvection in a suspension containing both nanoparticles and gyrotactic microorganisms. Int.Commun. Heat Mass Transfer 37, 14211425 .

Mabood, F. and K. Das (2016). Melting heat transfer on hydromagnetic flow of a nanofluid over a stretching sheet with radiation and second-order slip. European Physical Journal Plus 131 (1), 3 .

Mabood, F., S. Shateyi, M. M. Rashidi, E. Momoniat and N. Freidoonimehr (2016). MHD stagnation point flow heat and mass transfer of nanofluids in porous medium with radiation, viscous dissipation and chemical reaction. Advanced Powder Technology 27, 742-749.

Makinde, O. D. and A. Ogulu (2008). The effect of thermal radiation on the heat and mass transfer flow of a variable viscosity fluid past a vertical porous plate permeated by a transverse magnetic field. Chemical Engineering Communications 195 (12), 1575-1584.

Makinde, O., F. Mabood and M. Ibrahim (2018). Chemically reacting on MHD boundary layer flow of nanofluids over a non-linear stretching sheet with heat source/sink and thermal radiation. Thermal Science 22 (1), 495-506.

Morarji, D. V. and K. K. Gurjar (2019). Theoretical 
and Experimental Studies: $\mathrm{Cu}(\mathrm{I}) / \mathrm{Cu}$ (II) Catalytic Cycle in CuI/Oxalamide-promoted C-N bond formation. Organometallics 38(12), 2502-2511.

Mutuku, W. N. and O. D. Makinde (2014). Hydromagnetic bioconvection of nanofluid over a permeable vertical plate due to gyrotactic microorganisms. Computers \& Fluids. 95, 8897.

Pal, D. (2003). Hall current and MHD effects on heat transfer over an unsteady stretching permeable surface with thermal radiation. Computers \& Mathematics with Applications 66 (7), 11611180.

Pal, D. (2008). MHD Flow and heat transfer past a semi-infinite vertical plate embedded in a porous medium of variable Permeability. International Journal of Fluid Mechanics Research 35 (6), 493-509.

Pal, D. and P. Saha (2016). Influence of nonlinear thermal radiation and variable viscosity on hydromagnetic heat and mass transfer in a thin liquid film over an unsteady stretching surface. International Journal of Mechanical Sciences 119, 208-216.

Ramzan, M., J. D. Chung and U. Naeem (2017). Radiative magnetohydrodynamic nanofluid flow due to gyrotactic microorganisms with chemical reaction and non-linear thermal radiation. International Journal of Mechanical Sciences 130, 31-40.

Saidulu, N., T. Gangaiah and A. Lakshmi (2019). Impact of thermal radiation on MHD flow of tangent hyperbolic nanofluid over a nonlinear stretching sheet with convective boundary condition. Journal of Nanofluids 8 (1), 41-50.

Shahid, A., Z. Zhou1, M. M. Bhatti and D. Tripathi (2018). Magnetohydrodynamics nanofluid flow containing gyrotactic microorganisms propagating over a stretching Surface by Successive Taylor Series Linearization Method. Microgravity Science and Technology 30 (4), 445-455.

Syed, K. S., G. E. Tupholme and A. S. Wood (1997). Iterative solution of fluid flow in finned tubes. In Proceedings of the $10^{\text {th }}$ International Conference on Numerical Methods in Laminar and Turbulent Flow, Taylor C, Cross JT (eds). Pineridge Press: Swansea, U.K. 21-25, 429440.

Tausif, Sk. Md., D. Kalidas and P. K. Kundu (2016). Multiple slip effects on bioconvection of nanofluid flow containing gyrotactic microorganisms and nanoparticles. Journal of Molecular Liquids 220, 518-526.
Lakshmi, V. G., L. Anand Babu and K. Srinivasa Rao (2018). MHD mixed convection stagnation point flow of nanofluid through a porous medium over stretching sheet. International Journal of Pure and Applied Mathematics 118 (10), 369-389.

Waqas, H., S. U. Khan, S. A. Shehzad and M. Imran (2019). Radiative flow of Maxwell nanofluid containing gyrotactic microorganism and energy activation with convective Nield conditions. Heat Transfer - Asian Research 48, 1663-1687.

Watanabe, E., S. A. Chung, S. Nishimura, Y. Yamada, M. Okubo, K. Sodeyama, Y. Tateyama and A. Yamada (2019). Combined theoretical and experimental studies of sodium battery materials. The Chemical Record 19, 18.

$\mathrm{Xu}, \mathrm{H}$. and J. Cui (2018). Mixed convection flow in a channel with slip in a porous medium saturated with a nanofluid containing both nanoparticles and microorganisms. International Journal of Heat and Mass Transfer 125, 1043-1053.

Young, D. M. (1950). Iterative methods for solving partial differential equations of elliptic type. Doctoral Thesis, Harvard University Cambridge.

Zaib, A. M. M. Rashidi and A. J. Chamkha (2018). Flow of nanofluid containing gyrotactic microorganisms over static wedge in darcybrinkman porous medium with convective boundary condition. Journal of Porous Media 21(10), 911-928.

Zaimi, K., A. Ishak and I. Pop (2014). Stagnationpoint flow toward a stretchng/shrinking sheet in a nanofiuid containing both nanoparticies and gyrotactic microorganisms. Journal of Heat Transfer 136, 041705, 1-9.

Zheng, L., C. Zhang, X. Zhang and J. Zhang (2013) Radiation and heat transfer of a nanofluid over a stretching sheet with velocity slip and temperature jump in a porous medium. Journal of the Franklin Institute 350 (5), 990-1007.

Zhou, B., E. Bernhardt, A. Bhuyan, Z. Ghorbanishiadeh, N. Rasmussen, J. Lanska, and M. G. Kuzyk (2019). Theoretical and experimental studies of photomechanical materials [Invited]. Journal of the Optical Society of America B 36(6), 1492-1517.

Zuhra, S., N. S. Khan, Z. Shah, S. Islam and E. Bonyah (2018). Simulation of bioconvection in the suspension of second grade nanofluid containing nanoparticles and gyrotactic microorganisms. AIP ADVANCES 8, 1-23. 